\title{
Data Mining in Financial Markets
}

\author{
Longbing Cao \\ Associate Professor, the University of Technology, \\ Sydney (UTS), Australia
}

\begin{abstract}
The ongoing global financial recession has dramatically affected public confidence and market development. An example is the market manipulation schemes hidden in capital markets, which have caused losses in billions of dollars, dramatically damaging public confidence and contributing to the global financial and credit crisis. While most investors lost during market falls, for instance, sophisticated speculators can manipulate markets to make money by illegally using a variety of maneuvering techniques such as wash sales. With financial globalization, manipulators are becoming increasingly imaginative and professional, employing creative tactics such as using many nominee accounts at different broker-dealers. However, regulators currently are short on effective technology to promptly identify abnormal trading behavior related to complex manipulation schemes. As a result, shareholders are complaining that too few market manipulators were being caught. In this talk, I will discuss issues related to this topic, present case studies and lessons learned in identifying abnormal trading behavior in capital markets. I will discuss the use of data mining techniques in this area such as activity mining, combined mining, adaptive mining and domain-driven data mining.
\end{abstract}

